\title{
DIRICHLET PROBLEM FOR A NONLINEAR CONSERVATION LAW
}

\author{
Guy VALLET
}

\begin{abstract}
In this paper we propose the study of a first-order non-linear hyperbolic equation in a bounded domain. We give a result of existence and uniqueness of the entropic measure-valued solution and of the entropic weak solution; for some general assumptions on the data.
\end{abstract}

\section{Presentation of the model problem}

In this paper, we are concerned with a wide range of non-linear scalar conservation laws, arising from the mechanics of continua. Let us denote by $(P)$ the following problem :

$$
\begin{array}{cl}
\frac{\partial u}{\partial t}+\operatorname{div}(f(t, x, u))+g(t, x, u)=0 & \text { in } Q, \\
u=u^{B} & \text { on } \Sigma, \\
u=u_{0} & \text { on } \Omega,
\end{array}
$$

where :

-) $\Omega$ is a connected bounded open subset of $\mathbb{R}^{N}$, with a smooth boundary $\Gamma$, and an outward unit normal $\eta$,

-) $Q$ is the cylinder $] 0, T[\times \Omega, \Sigma$ its lateral boundary $] 0, T[\times \Gamma$ and $d i v=$ $\sum_{i=1}^{N} \frac{\partial}{\partial x_{i}}$

-) $H_{0}$ :

i) $u^{B}$ and $u_{0}$ are bounded measurable functions on $\Sigma$ and $\Omega$ respectively,

ii) $f$ is a continuous function on $\bar{Q} \times \mathbb{R}, g$ and $\operatorname{div} f$ are Caratheodory

1991 Mathematics Subject Classification: 35L60, 35L64, 35A05.

Servicio de Publicaciones. Universidad Complutense. Madrid, 2000 
functions,

iii) $\forall M>0, \exists g_{M} \in L^{2}(Q), \forall \lambda \in[-M, M]$,

$$
|g(., ., \lambda)| \leq g_{M} \text { and }|\operatorname{divf}(., ., \lambda)| \leq g_{M} \text { a.e. in } Q \text {. }
$$

It is well-known that, even if the data are regular, there is not, in general, a unique weak solution to a non-linear hyperbolic equation. One is then used to consider, among all the weak solutions, the one that satisfies an inequality called "entropy inequality".

We say that $u \in L^{\infty}(Q)$ is an entropic weak solution to the equation (1) if, for all positive $v$ of $H_{0}^{1}(Q)$ and all real $k$, one has :

$$
\begin{gathered}
\int_{Q} \operatorname{sign}(u-k)\left\{(u-k) \frac{\partial v}{\partial t}+[f(t, x, u)-f(t, x, k)] . \nabla v\right\} d x d t \\
-\int_{Q} \operatorname{sign}(u-k)\{\operatorname{div} f(t, x, k)+g(t, x, u)\} v d x d t \geq 0 .
\end{gathered}
$$

Since we do not suppose that $u_{0}$ belongs to $B V(\Omega) \cap L^{\infty}(\Omega)$,

i) Firstly, the usual compactness methods in $B V(Q) \cap L^{\infty}(Q)$ [2] seem to be deficient. Therefore, we consider here the framework of the measurevalued solutions [6] \& [12]. Moreover, it is known that the finite volume methods lead to some convergence in the sense of the Young measures. See for example the entropic process for the Cauchy problem [7], or, [5]\&[16] for bounded domains. Thus, the study of the uniqueness of such a solution, in a bounded domain, finds here all its interest.

ii) Secondly, $u$ does not belong to $B V(Q) \cap L^{\infty}(Q)$, so the trace of $u$ is not defined from the functional framework. However, as $u$ satisfies an entropy inequality, it is possible to consider some traces for $t=0$, as well as on $\Sigma$ (see the Young measure trace [11]). Thus, we will consider an entropy inequality including the initial and the boundary conditions, as introduced by F.Otto in [9] for $L^{\infty}$-functions.

Notations. In order to simplify the writing, let us put :

$$
\begin{gathered}
F(t, x, u, k)=\operatorname{sign}(u-k)[f(t, x, u)-f(t, x, k)], \\
G(t, x, u, k)=\operatorname{div} f(t, x, k)+g(t, x, u), \\
\mathfrak{L}(t, x, u, k, v)=|u-k| \frac{\partial v}{\partial t}+F(t, x, u, k) . \nabla v-\operatorname{sign}(u-k) G(t, x, u, k) v .
\end{gathered}
$$




\section{Basic properties of the Young measures}

We recall here some results concerning the Young measures, which can be found in [1], [6], [7], [12] and [13].

If $\mathcal{L}$ denotes the Lebesgue measure and $\mathbb{B}$ a bounded open subset of $\mathbb{R}^{m}$, then we have :

Definition 1. A positive Radon measure $\nu$ on $\mathbb{B} \times \mathbb{R}$ is a Young measure on $\mathbb{B} \times \mathbb{R}$ if for every Borel subset $A$ of $\mathbb{B}, \nu(A \times \mathbb{R})=\mathcal{L}(A)$. A Young measure $\nu$ is described by its disintegration form which is the unique family of probabilities on $\mathbb{R},\left(d P_{x}\right)_{x \in \mathbb{B}}$, such that for every Caratheodory function $\psi$,

$$
\int_{\mathbb{B} \times \mathbb{R}} \psi d \nu=\int_{\mathbb{B}} \int_{\mathbb{R}} \psi(x, \lambda) d P_{x}(\lambda) d x
$$

Example 1. We call Young measure associated to the measurable function $u$, the unique Young measure on $\mathbb{B} \times \mathbb{R}$, denoted by $\nu_{u}$, defined for every positive Caratheodory function $\psi$, by :

$$
\int_{\mathbb{B} \times \mathbb{R}} \psi d \nu_{u}=\int_{\mathbb{B}} \psi(x, u(x)) d x .
$$

That is, the disintegration form gives $d \nu(\lambda, x)=d \delta_{u(x)}(\lambda) d x$ where $\delta_{u(x)}$ denotes the Dirac mass centred in $u(x)$.

Proposition 1. (Gallouët) [7], [10]

Let $\nu$ be a Young measure on $\mathbb{B} \times \mathbb{R}$ and let $F_{x}$ be the repartition function of $d P_{x}$, the family of probabilities on $\mathbb{R}$ that is involved in the disintegration of $\nu$. Then, the function $u$, defined in $\mathbb{B} \times] 0,1[$ by

$$
u(x, \alpha)=\inf \left\{t \in \mathbb{R}, F_{x}(t)>\alpha\right\}
$$

is Lebesgue measurable on $\mathbb{B} \times] 0,1[$ and for every positive Caratheodory function $\psi$,

$$
\int_{\mathbb{B} \times \mathbb{R}} \psi d \nu=\int_{\mathbb{B}} \int_{\mathbb{R}} \psi(x, \lambda) d P_{x}(\lambda) d x=\int_{\mathbb{B}} \int_{0}^{1} \psi(x, u(x, \alpha)) d \alpha d x .
$$

Moreover, if $\operatorname{Supp}\left(d P_{x}\right) \subset[-M, M]$, then $u \in L^{\infty}(\mathbb{B} \times] 0,1[)$ and $\|u\|_{\infty} \leq$ $M$.

$u$ is called the process associated to the Young measure $\nu$. 
Proposition 2. Let $\left(u_{n}\right)_{n}$ be a sequence of $L^{\infty}(\mathbb{B})$ functions such that $\forall n \in \mathbb{N},\left\|u_{n}\right\|_{\infty} \leq M$. Then, there exists a sub-sequence $\left(u_{n_{k}}\right)_{k}$ and a Young measure $\nu\left(\nu=d P_{x} d x\right)$ on $\mathbb{B} \times \mathbb{R}$ with $\operatorname{Supp}\left(d P_{x}\right) \subset[-M, M]$, such that for every $L^{1}\left(\mathbb{B}, \mathcal{C}_{b}(\mathbb{R})\right)$ function $\psi$,

$$
\int_{\mathbb{B}} \psi\left(x, u_{n}(x)\right) d x \rightarrow \int_{\mathbb{B} \times \mathbb{R}} \psi(x, \lambda) d \nu .
$$

Futhermore, if the limit $\nu$ is the Young measure associated to $u$, then $u_{n_{k}}$ converges towards $u$ in $L^{p}(\mathbb{B}), 1 \leq p<\infty$.

Remark 1. In [1], E. J. Balder defines the Young measures and gives the same kind of results with $(\mathbb{B}, \mathcal{B}, \mathcal{L})$ being a finite (or $\sigma$-finite) measured space. This definition allows us to talk about Young measure trace on a hypersurface of $\mathbb{R}^{m}$, provided with the Hausdorff measure $\mathcal{H}^{m-1}$.

\section{Definition of an Entropic Measure-Valued So- lution}

Definition 2. A Young measure $\nu$ is an Entropic Measure-Valued Solution (emvs) to (1) if :

i) $\exists M>0, \operatorname{Supp}\left(d P_{(t, x)}\right) \subset[-M, M]$ where $d \nu(t, x, \lambda)=d P_{(t, x)}(\lambda) d x d t$. ii) $\forall v \in H_{0}^{1}(Q), v \geq 0, \forall k \in \mathbb{R}, \int_{Q \times \mathbb{R}} \mathfrak{L}(t, x, \lambda, k, v) d \nu \geq 0$.

Proposition 3. Let $\nu$ be an emvs to (1). Then, for any positive $\beta$ of $L^{1}(\Omega)$ and any $w$ in $L^{\infty}(\Omega)$,

$$
\lim _{t \rightarrow 0^{+}} \operatorname{ess} \int_{\Omega \times \mathbb{R}}|\lambda-w(x)| \beta(x) d P_{(t, x)}(\lambda) d x \text { exists. }
$$

Proof. Consider in definition $2 i i), v(t, x)=\alpha(t) \beta(x), \alpha \in \mathcal{D}^{+}(0, T)$, $\beta \in \mathcal{D}^{+}(\Omega)$. Then it comes that :

$$
\begin{gathered}
0 \leq \int_{0}^{T} \alpha^{\prime}(t) \int_{\Omega \times \mathbb{R}}|\lambda-k| \beta(x) d P_{(t, x)}(\lambda) d x d t+ \\
\int_{0}^{T} \alpha(t) \int_{\Omega \times \mathbb{R}} F(t, x, \lambda, k) \nabla \beta-\operatorname{sign}(\lambda-k) G(t, x, \lambda, k) \beta d P_{(t, x)}(\lambda) d x d t .
\end{gathered}
$$


Let us note $h^{k, \beta}(t)=\int_{\Omega \times \mathbb{R}}|\lambda-k| \beta(x) d P_{(t, x)}(\lambda) d x$. As $h^{k, \beta}$ is an essentially bounded function, the above inequality shows that $h^{k, \beta}$ is a.e. a bounded variation function. So, one can talk about $\lim _{t \rightarrow 0^{+}} e s h^{k, \beta}(t)$.

As, uniformly with respect to $t, \beta \mapsto h^{k, \beta}(t)$ is a continuous linear functional on $L^{1}(\Omega)$, a density argument leads to the existence, for any $k$ in $\mathbb{R}$ and any positive $\beta$ in $L^{1}(\Omega)$, of

$$
\lim _{t \rightarrow 0^{+}} \operatorname{ess} \int_{\Omega \times \mathbb{R}}|\lambda-k| \beta(x) d P_{(t, x)}(\lambda) d x .
$$

In order to work with an essential limit in the sequel, we only consider $k$ in $\mathbb{Q}$.

Let $w_{n}=\sum_{i=0}^{n} k_{i} \mathbb{I}_{B_{i}}$ be a simple function on $\Omega$ with $k_{i}$ belonging to $\mathbb{Q}$. As, $\int_{\Omega \times \mathbb{R}}\left|\lambda-w_{n}(x)\right| \beta(x) d P_{(t, x)}(\lambda) d x=\sum_{i=0}^{n} \int_{\Omega \times \mathbb{R}}\left|\lambda-k_{i}\right| \beta(x) \mathbb{I}_{B_{i}} d P_{(t, x)}(\lambda)$ $d x, \lim _{t \rightarrow 0^{+}} \operatorname{ess}^{w, \beta}(t)$ exists for any positive $\beta$ in $L^{1}(\Omega)$ and any' $\mathbb{Q}$-valued simple function $w$ on $\Omega$.

As any $w$ in $L^{\infty}(\Omega)$ is a limit in $L^{\infty}(\Omega)$ of a sequence of such simple functions and since for $w$ and $\widehat{w}$ in $L^{\infty}(\Omega),\left|h^{w, \beta}(t)-h^{\widehat{w}, \beta}(t)\right| \leq|\beta|_{L^{1}} \cdot \mid w-$ $\left.\widehat{w}\right|_{L^{\infty}}$, independently upon $t$, the same argument of density leads to the result.

Definition 3. An emvs to (1) satisfies the initial condition (3) if

$$
\lim _{t \rightarrow 0^{+}} \operatorname{ess} \int_{\Omega \times \mathbb{R}}\left|\lambda-u_{0}(x)\right| d P_{(t, x)}(\lambda) d x=0 .
$$

Definition 4. (Szepessy [11]) Let us introduce, for $x$ in the neighborhood of $\Gamma$, the change of coordinates $x \rightarrow(\bar{x}, \xi): x=\bar{x}-\xi . \eta(\bar{x})$ where $(\bar{x}, \xi) \in \Gamma \times(0, \varepsilon)$ for some positive $\varepsilon, \eta$ is the unit outward normal, and $J(\bar{x}, \xi)$ is the jacobian determinant associated to this change of coordinates.

Then, there is a sequence $\xi_{i}$ in $(0, \varepsilon), \xi_{i} \rightarrow 0^{+}$and a Young measure $\mu$ on $\Sigma \times \mathbb{R}$, described by its disintegration form : $d \mu(\lambda, t, x)=d P_{(t, x)}^{\mu}(\lambda) d \mathcal{H}^{N}$, where $\mathcal{H}^{N}$ is the $N$ dimensional Hausdorff measure on $\Sigma$ and where $d P_{(t, x)}^{\mu}(\lambda)$ is a family of probabilities on $\mathbb{R}$, satisfying the point $\left.i\right)$ of definition 2, such that : for any function $\psi$ in $L^{1}\left(\Sigma, C_{b}(\mathbb{R})\right)$, the next 
relation holds :

$$
\operatorname{Lim}_{j \rightarrow \infty} \int_{\Sigma} \int_{\mathbb{R}} \psi(t, \bar{x}, \lambda) d P_{\left(t, x\left(\bar{x}, \xi_{j}\right)\right.}(\lambda) J\left(\bar{x}, \xi_{j}\right) d \mathcal{H}^{N}=\int_{\Sigma} \int_{\mathbb{R}} \psi(t, \bar{x}, \lambda) d \mu .
$$

$\mu$ is called a Young measure trace on $\Sigma$ for $\nu$.

Using this trace, we prove :

Proposition 4. Let $\nu$ be an emvs to (1) and $\mu$ a Young measure trace for $\nu$. Then, for any positive $\alpha$ of $L^{1}(\Sigma)$ and any $w$ in $L^{\infty}(\Sigma)$, one has:

$$
\begin{gathered}
\lim _{\xi \rightarrow 0^{+}} e s s \int_{\Sigma \times \mathbb{R}} F(t, x, \lambda, w) \cdot \eta(\bar{x}) \alpha(t, \bar{x}) d P_{(t, x)}(\lambda) J d \mathcal{H}^{N}= \\
\int_{\Sigma \times \mathbb{R}} F(t, x, \lambda, w) \cdot \eta \alpha d \mu
\end{gathered}
$$

Proof. Consider in definition $2 i i), v(t, x)=\alpha(t, \bar{x}) \beta(\xi), \alpha \in \mathcal{D}^{+}(\Sigma)$, $\beta \in \mathcal{D}^{+}(0, \varepsilon)$. Then,

$$
\begin{gathered}
0 \leq-\int_{0}^{\varepsilon} \beta^{\prime}(\xi) \int_{\Sigma \times \mathbb{R}} F(t, x, \lambda, k) \cdot \eta(\bar{x}) \alpha(t, \bar{x}) d P_{(t, x)}(\lambda) J d \mathcal{H}^{N} d \xi+ \\
\int_{0}^{\varepsilon} \beta(\xi) \int_{\Sigma \times \mathbb{R}}|\lambda-k| \frac{\partial \alpha}{\partial t} d P_{(t, x)}(\lambda) J d \mathcal{H}^{N} d \xi+ \\
\int_{0}^{\varepsilon} \beta(\xi) \int_{\Sigma \times \mathbb{R}} F(t, x, \lambda, k) \nabla \alpha-\operatorname{sign}(\lambda-k) G(t, x, \lambda, k) \alpha J d P_{(t, x)}(\lambda) d \mathcal{H}^{N} d \xi .
\end{gathered}
$$

Thus, for $h^{k, \alpha}(\xi)=\int_{\Sigma \times \mathbb{R}} F(t, x, \lambda, k) \cdot \eta(\bar{x}) \alpha(t, \bar{x}) J d P_{(t, x)}(\lambda) d \mathcal{H}^{N}$, the same argument as in the previous proposition leads to the existence of $\lim _{\xi \rightarrow 0^{+}} e s s h^{w, \alpha}(\xi)$, for any positive $\alpha$ in $L^{1}(\Sigma)$ and any $\mathbb{Q}$-valued simple function $w$ on $\Sigma$. Since $f$ is a continuous function, $\lim _{\xi \rightarrow 0^{+}} \operatorname{essh}^{w, \alpha}(\xi)=$ $\int_{\Sigma \times \mathbb{R}} F(t, \bar{x}, \lambda, w) \cdot \eta(\bar{x}) \alpha(t, \bar{x}) d \mu$.

Let $w$ be in $L^{\infty}(\Sigma)$ and let $w_{n}=\sum_{i=0}^{n} k_{i} \mathbb{I}_{B_{i}}$ be a simple function on $\Sigma$, with $k_{i} \in \mathbb{Q}$, that converges to $w$ in $L^{\infty}(\Sigma)$.

As $f$ is continuous on $\bar{Q} \times \mathbb{R}$ and as $w_{n}$ converges in $L^{\infty}(\Sigma), \mid h^{w, \alpha}(\xi)-$ $h^{w_{n}, \alpha}(\xi) \mid$ converges to 0 uniformly with respect to $\xi$ as $n$ goes to infinity. 
So, $\lim _{\xi \rightarrow 0^{+}} \operatorname{ess}^{w, \alpha}(\xi)=\int_{\Sigma \times \mathbb{R}} F(t, x, \lambda, w) \cdot \eta(\bar{x}) \alpha(t, \bar{x}) d \mu$ can be extended to any positive $\alpha$ of $L^{1}(\Sigma)$ and any $w$ in $L^{\infty}(\Sigma)$.

Following Felix Otto's idea in [9], we will say that :

Definition 5. An emvs of (1) satisfies the boundary condition (2) if for a Young measure trace $\mu$, any real $k$ and any positive $\alpha$ of $L^{1}(\Sigma)$ :

$$
\begin{gathered}
\int_{\Sigma} F\left(t, x, k, u^{B}\right) \cdot \eta \alpha d \mathcal{H}^{N} \leq \\
\int_{\Sigma \times \mathbb{R}} F\left(t, x, \lambda, u^{B}\right) \cdot \eta \alpha d \mu+\int_{\Sigma \times \mathbb{R}} F(t, x, \lambda, k) \cdot \eta \alpha d \mu .
\end{gathered}
$$

Remark 2. If $\nu$ is a Young measure associated to a function $u$ and if there exists a trace $\gamma(u)$ in $L^{\infty}(\Sigma)$ for $u$, then $d P_{(t, x)}^{\mu}=\delta_{\gamma(u(t, x))}$ and the boundary condition is equivalent to

$$
F(t, x, \gamma(u), k) . \eta(x) \geq 0, \forall k \in\left[\min \left(\gamma(u), u^{B}\right), \max \left(\gamma(u), u^{B}\right)\right] .
$$

This condition is exactly the one given in [2].

Definition 6. A Young measure $\nu$ is an Entropic Measure-Valued Solution to the problem $(P)$ (EMVS) if it is an emvs to (1) satisfying the initial and the boundary conditions, as mentioned above in definitions 3 and 5 .

Proposition 5. Let $\nu$ be an EMVS to $(P)$ and $\mu$ a Young measure trace, then for any positive $\beta$ in $H^{1}(Q)$ and any $k$ in $\mathbb{R}$,

$$
\begin{gathered}
-\int_{Q \times \mathbb{R}} \mathfrak{L}(t, x, \lambda, k, \beta) d \nu \leq \int_{\Omega}\left|u_{0}-k\right| \beta(0) d x+ \\
\int_{\Sigma \times \mathbb{R}} F\left(t, x, \lambda, u^{B}\right) \cdot \eta \beta d \mu-\int_{\Sigma} F\left(t, x, k, u^{B}\right) \cdot \eta \beta d \mathcal{H}^{N} .
\end{gathered}
$$

Proof. Following [15], let $\beta$ be in $\mathcal{D}^{+}(\bar{Q}), \varphi_{n}(t)=\max (0, \min (n t, n(T-$ $t), 1)), \alpha_{n}(x)=\min (n \xi, 1)$ and consider in definition 2 ii) 
$v=\beta(t, x) \cdot \alpha_{n}(x) \cdot \varphi_{n}(t)$. By passing $n$ to the limit in the inequality $\int_{Q \times \mathbb{R}} \mathfrak{L}\left(t, x, \lambda, k, \beta \alpha_{n} \varphi_{n}\right) d \nu \geq 0$ one has,

$$
\begin{gathered}
-\int_{Q \times \mathbb{R}} \mathfrak{L}(t, x, \lambda, k, \beta) d \nu \leq \\
\int_{\Omega}\left|u_{0}-k\right| \beta(0) d x-\int_{\Sigma \times \mathbb{R}} F(t, x, \lambda, k) \cdot \eta \beta d \mu
\end{gathered}
$$

for any positive function $\beta$ of $\mathcal{D}(\bar{Q})$ (and for any positive function $\beta$ of $H^{1}(Q)$ by density).

To conclude, one has simply to use the definition 5 .

\section{Existence of an Entropic Measure-Valued So- lution}

In order to prove the existence of an EMVS, we consider the same hyperbolic problem, with some regular data $f_{\varepsilon}, g_{\varepsilon}, u_{\varepsilon}^{B}$ and $u_{0, \varepsilon}$; and we study the limit when $\varepsilon$ goes to 0 . This kind of problem is well known and has been studied in [2] and [8].

Let us give below the assumptions needed for the existence of a solution $\left(H_{1}\right)$ :

i) $H_{0}$ (cf. first paragraph),

ii) $\exists c_{1}, c_{2} \in L^{\infty}(Q), \forall \lambda \in \mathbb{R},(t, x)$ a.e. in $Q$,

$$
|g(t, x, \lambda)| \leq c_{1}(t, x)|\lambda|+c_{2}(t, x)
$$

with $c_{i}(g)=\left\|c_{i}\right\|_{\infty} i=1,2$,

iii) $f$ can be continuously prolonged on $\left(\mathbb{R}^{N+1} \backslash Q\right) \times \mathbb{R}$ by a function still denoted by $f$ and $\exists c_{1}^{\prime}, c_{2}^{\prime} \in L^{\infty}\left(\mathbb{R}^{N}\right), \forall \lambda \in \mathbb{R},(t, x)$ a.e. in $\mathbb{R}^{N+1}$,

$$
|\operatorname{divf}(t, x, \lambda)| \leq c_{1}^{\prime}(t, x)|\lambda|+c_{2}^{\prime}(t, x),
$$

with $c_{i}(f)=\left\|c_{i}^{\prime}\right\|_{\infty} i=1,2$.

For $\epsilon>0$, let us consider the $B V(Q) \cap L^{\infty}(Q)$ solution $u_{\epsilon}$ of the hyperbolic problem $\left(P_{\varepsilon}\right)$ :

$$
\frac{\partial u_{\epsilon}}{\partial t}+\operatorname{div}\left(f_{\epsilon}\left(t, x, u_{\epsilon}\right)\right)+g_{\epsilon}\left(t, x, u_{\epsilon}\right)=0 \quad \text { in } Q
$$




$$
u_{\epsilon}=u_{\epsilon}^{B} \text { on } \Sigma ; \text { and } u_{\epsilon}=u_{0, \epsilon} \text { on } \Omega,
$$

where $f_{\epsilon}(t, x, \lambda)=\int_{\mathbb{R}} \int_{\mathbb{R}^{N+1}} f(s, y, \xi) \rho_{1}^{n}(\lambda-\xi) \rho_{N+1}^{n}((t, x)-(s, y)) d s d y d \xi$, $\rho_{1}^{n}$ and $\rho_{N+1}^{n}$ being the standard mollifier sequences respectively in $\mathbb{R}$ and $\mathbb{R}^{N+1}, g_{\epsilon}$ is defined in the same way and $u_{\epsilon}^{B}$ and $u_{0, \epsilon}$ are regularisations of the boundary and initial data of the problem $(P)$, bounded by the same constant.

Proposition 6. There exists a unique entropic weak solution $u_{\epsilon}$ to this problem; moreover,

i) $u_{\epsilon}$ is essentially bounded, independently of $\epsilon$, by :

$$
M=\frac{K_{2}}{K_{1}}\left(e^{K_{1} T}-1\right)+\left(\left\|u_{0}\right\|_{\infty}+\left\|u^{B}\right\|_{\infty}\right) e^{K_{1} T}
$$

where $K_{i}=c_{i}(f)+c_{i}(g), i=1,2$.

ii) $u_{\varepsilon}$ is also a solution, for any positive $\mathcal{C}^{2}$ convex function $\theta$ with $\theta(0)=\theta^{\prime}(0)=0$, any positive $\beta$ of $H_{0}^{1}(Q)$ and any $k$ in $\mathbb{R}$, of the inequality :

$$
\begin{gathered}
\int_{Q} \theta\left(u_{\varepsilon}-k\right) \frac{\partial \beta}{\partial t}+\left[\theta^{\prime}\left(u_{\varepsilon}-k\right) f_{\varepsilon}\left(t, x, u_{\varepsilon}\right)-\int_{k}^{u_{\varepsilon}} \theta^{\prime \prime}(\xi-k) f_{\varepsilon}(t, x, \xi) d \xi\right] . \nabla \beta- \\
{\left[\int_{k}^{u_{\varepsilon}} \theta^{\prime \prime}(\xi-k) \operatorname{div} f_{\varepsilon}(t, x, \xi) d \xi+\theta^{\prime}\left(u_{\varepsilon}-k\right) g_{\varepsilon}\left(t, x, u_{\varepsilon}\right)\right] \beta d x d t \geq 0 .}
\end{gathered}
$$

Proof. This result is classical in $B V(Q) \cap L^{\infty}(Q)$ (see [2] and [8] for i).

Since $u_{\varepsilon}$ is bounded in $L^{\infty}(Q)$, it is possible to extract a subsequence which converges to a Young measure $\nu$ (in the sense of proposition 2). Moreover, $\nu$ satifies the assumption $i$ ) of definition 2.

So, for any positive $\mathcal{C}^{2}$ convex function $\theta$ with $\theta(0)=\theta^{\prime}(0)=0$, for any positive $\beta$ of $H_{0}^{1}(Q)$ and any $k$ in $\mathbb{R},(5)$ leads to :

$$
\begin{gathered}
\int_{Q}\left\{\theta(\lambda-k) \frac{\partial \beta}{\partial t}+\left[\theta^{\prime}(\lambda-k) f(t, x, \lambda)-\int_{k}^{\lambda} \theta^{\prime \prime}(\xi-k) f(t, x, \xi) d \xi\right] . \nabla \beta-\right. \\
\left.\left[\int_{k}^{\lambda} \theta^{\prime \prime}(\xi-k) \operatorname{divf}(t, x, \xi) d \xi+\theta^{\prime}(\lambda-k) g(t, x, \lambda)\right] \beta\right\} d \nu \geq 0 .
\end{gathered}
$$

Now, considering $\theta_{n}(\xi)=\int_{0}^{\xi} \int_{0}^{t}\left[\rho_{n}(s-1 / n)+\rho_{n}(s+1 / n)\right] d s d t$, one has: 
Proposition 7. There exists an emvs to equation (1).

Proposition 8. This emvs is an EMVS to the problem $(P)$.

Proof. Remark that the Young measure associated to the weak solution $u_{\varepsilon}$ (see example 1) is also an EMVS to problem $\left(P_{\varepsilon}\right)$ in the sense of definition 6. Thus, as mentioned in inequation (1), for any positive function $\beta$ in $H^{1}(Q)$,

$$
\begin{gathered}
-\int_{Q} \mathfrak{L}_{\varepsilon}\left(t, x, u_{\varepsilon}, k, \beta\right) d x d t \leq \\
\int_{\Omega}\left|u_{0, \varepsilon}-k\right| \beta(0) d x-\int_{\Sigma} F_{\varepsilon}\left(t, x, u_{\varepsilon}, k\right) \cdot \eta \beta d \mathcal{H}^{N} .
\end{gathered}
$$

Let us make $\varepsilon$ go to 0 , then :

$$
\begin{gathered}
-\int_{Q \times \mathbb{R}}|\lambda-k| \frac{\partial \beta}{\partial t}+F(t, x, \lambda, k) \cdot \nabla \beta-\chi_{k}(t, x) \beta d \nu \leq \\
\int_{\Omega}\left|u_{0}-k\right| \beta(0) d x-\int_{\Sigma \times \mathbb{R}} F(t, x, \lambda, k) \cdot \eta \beta d \widetilde{\mu}
\end{gathered}
$$

where $\chi_{k}$ is the $L^{\infty}(Q) *$ limit of $\operatorname{sign}\left(u_{\varepsilon}-k\right) G_{\varepsilon}\left(t, x, u_{\varepsilon}, k\right)$ and $\tilde{\mu}$ the Young measure on $\Sigma \times \mathbb{R}$, limit of $\left(\operatorname{trace}\left(u_{\varepsilon}\right)\right)_{\varepsilon}$ in the sense of proposition 2 (see the remark that follows this proposition and also [1]).

Firstly, consider $\beta(t, x)=\alpha(x) \cdot \min \left((1-n t)^{+}, 1\right)$.

Since $\lim _{t \rightarrow 0^{+}} \operatorname{ess} \int_{\Omega \times \mathbb{R}}|\lambda-k| \alpha d P_{(t, x)}(\lambda) d x \leq \int_{\Omega}\left|u_{0}-k\right| \alpha d x$, replacing $k$ by $u_{0}$ as shown in the demonstration of proposition 3 , implies that $\nu$ satifies the initial condition.

Secondly, consider $\beta(t, x)=\alpha(t, x) \cdot \min \left((1-n \xi)^{+}, 1\right)$ with the variables introduced in definition 4 ( i.e. $x=\bar{x}-\xi \eta(\bar{x})$ ). As

$$
\int_{\Sigma \times \mathbb{R}} F(t, x, \lambda, k) \cdot \eta(\bar{x}) \alpha(t, \bar{x}) d \mu \geq \int_{\Sigma \times \mathbb{R}} F(t, x, \lambda, k) \cdot \eta(\bar{x}) \alpha(t, \bar{x}) d \widetilde{\mu},
$$

replacing $k$ by $u^{B}$ as shown in the demonstration of proposition 4 , gives that

$$
\int_{\Sigma \times \mathbb{R}} F\left(t, x, \lambda, u^{B}\right) \cdot \eta(\bar{x}) \alpha(t, \bar{x}) d \mu \geq \int_{\Sigma \times \mathbb{R}} F\left(t, x, \lambda, u^{B}\right) \cdot \eta(\bar{x}) \alpha(t, \bar{x}) d \widetilde{\mu} .
$$


Since $u_{\varepsilon}$ is a solution of $\left(P_{\varepsilon}\right)$, it satisfies the boundary condition :

$$
F_{\varepsilon}\left(t, x, k, u_{\varepsilon}^{B}\right) \cdot \eta \leq F_{\varepsilon}\left(t, x, u_{\varepsilon}, k\right) \cdot \eta+F_{\varepsilon}\left(t, x, u_{\varepsilon}, u_{\varepsilon}^{B}\right) \cdot \eta \quad \text { a.e. on } \Sigma
$$

Then, since $\alpha$ is a positive function, let us next pass to the limit in (8) as $\varepsilon$ goes to 0 . The upper semi-continuous properties (6) and (7) of the trace imply that $\nu$ satisfies the boundary condition.

\section{Uniqueness of the Entropic Measure - Valued Solution to Problem (P) and existence of a weak solution}

In many non linear problems, compactness results are essential in order to obtain the existence of solutions. Usually, one manages to extract a sub-sequence that converges a.e. in order to identify the limits of the non linear terms. When $L^{1}$ sequencial compactness fails, one looks for Young measure solutions (see [6], [12], [9], [8] and [14] for the study of hyperbolic equations, or [7], [4], [3], [5], [11] and [16] for numerical estimations).

For non linear hyperbolic equations, thanks to the uniqueness result for entropic measure valued solutions, it is possible to prove that the Young measure solution is associated to a function. This function is then the weak entropic solution. Moreover, proposition 2 transform the weak convergence of $\left(u_{\varepsilon}\right)_{\varepsilon}$ (see the previous paragraph) into a strong convergence in $L^{p}$, for all finite $p$. It is therefore important to have a result of uniqueness of measure-valued solutions, this result leading to the existence (and the uniqueness) of a weak solution.

We say that a measurable function $h$ on $Q \times \mathbb{R}$ satisfies the property $(\Re)$ if

i) $\forall M>0, \exists g_{M} \in L^{\infty}(Q), \forall \lambda \in[-M, M],|h(., ., \lambda)| \leq g_{M}$ a.e. in $Q$,

ii) $\forall(x, t)$ a.e. in $Q, \forall \varepsilon>0, \exists \eta>0,|\alpha-\beta| \leq \eta \Rightarrow|h(t, x, \alpha)-h(t, x, \beta)| \leq$ $\varepsilon$.

Let us give below the assumptions needed for the uniqueness of the solution $\left(\mathrm{H}_{2}\right)$ :

i) $H_{0}$ (cf. first paragraph),

ii) Assume that $t \mapsto f(t, x, \lambda)$ and $x_{i} \mapsto f(t, x, \lambda), i \in\{1, . ., N\}$ are absolutely continuous functions (i.e. for a fixed $\lambda, f(., ., \lambda) \in W^{1,1}(Q)$ ) 
and $\frac{\partial f}{\partial t}$ and $\frac{\partial f}{\partial x_{i}}, \forall i \in\{1, \ldots, N\}$, satisfy property $(\Re)$, iii) a) $g$ satisfies property $(\Re)$ and

b) $\exists c \in L^{\infty}(Q),|g(t, x, \alpha)-g(t, x, \beta)| \leq c(t, x)|\alpha-\beta| \forall \alpha, \beta \in$ $\mathbb{R},(t, x)$ a.e. in $Q$, with $c(g)=\|c\|_{\infty}$.

Remark 3. $\left.H_{2}, i i i\right) b$ ) entails property $(\Re)$ if $g(., ., 0) \in L^{\infty}(Q)$; $\operatorname{divf}$ satisfies property $(\Re)$ because $\frac{\partial f}{\partial x_{i}}, \forall i \in\{1, . ., N\}$ satisfy it.

Proposition 9. (Krushkov inequality) Assume that $\nu$ and $\widehat{\nu}$ are two admissible EMVS to problem $(P)$ with two initial conditions $u_{0}$ and $\hat{u}_{0}$ and two boundary conditions $u^{B}$ and $\hat{u}^{B}$, and let us note $d \nu(\lambda, t, x)=$ $d P_{(t, x)}(\lambda) d x d t$ and $d \widehat{\nu}(\lambda, t, x)=d \widehat{P}_{(t, x)}(\lambda) d x d t$. Then for $t$ a.e. in ] $0, T[:$

$$
\begin{gathered}
\int_{\Omega \times \mathbb{R}^{2}}|\lambda-k| d P_{(t, x)}(\lambda) d \widehat{P}_{(t, x)}(k) d x+ \\
\int_{0}^{t} \int_{\Omega \times \mathbb{R}^{2}} \operatorname{Sgn}(\lambda-k)[g(s, x, \lambda)-g(s, x, k)] d P_{(s, x)}(\lambda) d \widehat{P}_{(s, x)}(k) d x d s \leq \\
\int_{0}^{t} \int_{\Gamma} \Delta\left(s, x, u^{B}, \hat{u}^{B}\right) d \mathcal{H}^{N-1} d s+\int_{\Omega}\left|u_{0}-\hat{u}_{0}\right| d x \\
\text { where } \Delta(t, x, A, B)=\underset{\operatorname{Sup}}{\operatorname{Sup}(A, B) \leq c, d \leq \max (A, B)}|f(t, x, c)-f(t, x, d)| .
\end{gathered}
$$

Proof. Let us note $\beta(t, s, x, y)=\gamma(t) \rho_{1}^{n}(t-s) \rho_{N}^{n}(x-y)$ where $\gamma$ is a positive function of $\mathcal{D}(] 0, T[)$ and $\rho_{1}^{n}$ and $\rho_{N}^{n}$ are the standard mollifier functions indexed by $n$. Then,

$$
\begin{gathered}
-\int_{(Q \times \mathbb{R})^{2}}|\lambda-k| \gamma^{\prime}(t) \rho_{1}^{n}(t-s) \rho_{N}^{n}(x-y) d \nu(t, x, \lambda) d \widehat{\nu}(s, y, k)+ \\
\int_{(Q \times \mathbb{R})^{2}} S g n(\lambda-k)[G(t, x, \lambda, k)-G(s, y, k, \lambda)] \gamma(t) \rho_{1}^{n}(t-s) \rho_{N}^{n}(x-y) d \nu d \widehat{\nu}- \\
\int_{(Q \times \mathbb{R})^{2}} \gamma(t) \rho_{1}^{n}(t-s)[F(t, x, \lambda, k)-F(s, y, k, \lambda)] \cdot \nabla_{x} \rho_{N}^{n}(x-y) d \nu d \widehat{\nu} \leq \\
\int_{\Omega \times Q \times \mathbb{R}}\left|\hat{u}_{0}(y)-\lambda\right| \gamma(t) \rho_{1}^{n}(t) \rho_{N}^{n}(x-y) d y d \nu(t, x, \lambda)+ \\
\int_{\Sigma \times \mathbb{R} \times Q \times \mathbb{R}} F\left(t, x, \lambda, u^{B}\right) \cdot \eta \gamma(t) \rho_{1}^{n}(t-s) \rho_{N}^{n}(x-y) d \mu d \widehat{\nu}(s, y, k)+
\end{gathered}
$$


GUY VALLET DIRICHLET PROBLEM FOR A NONLINEAR CONSERVATION LAW ...

$$
\begin{gathered}
\int_{\Sigma \times \mathbb{R} \times Q \times \mathbb{R}} F\left(s, y, k, \hat{u}^{B}\right) \cdot \eta \gamma(t) \rho_{1}^{n}(t-s) \rho_{N}^{n}(x-y) d \widehat{\mu} d \nu(t, x, \lambda)- \\
\int_{\Sigma \times Q \times \mathbb{R}} F\left(t, x, k, u^{B}\right) \cdot \eta \gamma(t) \rho_{1}^{n}(t-s) \rho_{N}^{n}(x-y) d \mathcal{H}^{N} d \widehat{\nu}(s, y, k)- \\
\int_{\Sigma \times Q \times \mathbb{R}} F\left(s, y, \lambda, \hat{u}^{B}\right) \cdot \eta \gamma(t) \rho_{1}^{n}(t-s) \rho_{N}^{n}(x-y) d \mathcal{H}^{N} d \nu(t, x, \lambda) .
\end{gathered}
$$

In order to pass to the limit in (9) as $n$ goes to infinity, let us denote by $I_{1}, I_{2}, I_{3}, I_{4}, I_{5}, I_{5}$ bis,$I_{6}$ and $I_{6 \text { bis }}$ each of the previous integrals. Thus (9) is equivalent to : $-I_{1}+I_{2}-I_{3} \leq I_{4}+I_{5}+I_{5 \text { bis }}-I_{6}-I_{6 \text { bis }}$.

According to the notations of Th. Gallouët (cf. proposition 1 ), we will note $u(t, x, \alpha)$ the entropic process associated to $\nu$ and $\hat{u}(s, y, \beta)$ the one associated to $\widehat{\nu}$. Finally, let us note $A_{n}(t, s, x, y)=\rho_{1}^{n}(t-s) \rho_{N}^{n}(x-y)$.

The study of the limit in (9) will be done in seven steps.

Step 1. $\lim _{n \rightarrow \infty} I_{1}=\int_{Q} \gamma^{\prime}(t) \int_{\mathbb{R}^{2}}|\lambda-k| d P_{(t, x)}(\lambda) d \widehat{P}_{(t, x)}(k) d x d t$.

Proof. See [7] and [15].

Lemma 10. Assume that $h$ is a measurable function on $Q \times \mathbb{R}$ that satisfies property $(\mathfrak{R})$. Then,

i) $\int_{\left.Q^{2} \times\right] 0,1[}\left\{|h(s, y, u(s, y, \alpha))-h(s, y, u(t, x, \alpha))| \gamma(t) A_{n}\right\} d \alpha d x d t d y d s$ converges towards 0 as $n$ goes to infinity (Resp. with $\hat{u}$ ).

ii) Let us note $H(t, x, a, b)=\operatorname{sign}(a-b)[h(t, x, a)-h(t, x, b)]$ then

$$
\begin{aligned}
& \int_{(Q \times] 0,1[)^{2}} \gamma(t) A_{n}\{H(t, x, u(t, x, \alpha), \hat{u}(s, y, \beta))- \\
& H(t, x, u(t, x, \alpha)-\hat{u}(t, x, \beta))\} d \alpha d \beta d x d t d y d s
\end{aligned}
$$

converges towards 0 as $n$ goes to infinity.

Proof. [15] Since $h$ satisfies property $(\Re)$ and since the functions $u$ and $\hat{u}$ are bounded, one can suppose that $h$ is bounded by a $L^{\infty}(Q)$ function.

The relation i) follows from the property $(\Re)$ and from the fact that there exists a sequence of simple functions $u_{k}=\sum_{i=1}^{k} k_{i} \mathbb{I}_{B_{i}}$, converging towards $u$ in $L^{\infty}(Q \times \mathbb{R})$ with $\left\|u_{k}\right\|_{\infty} \leq\|u\|_{\infty}$. 
Point ii) follows from Lusin theorem that allows us to replace $\hat{u}$ by a continuous function, excepted for a set with small Lebesgue measure.

Step 2. $\lim _{n \rightarrow \infty} I_{2}=$ $\int_{Q \times \mathbb{R}^{2}} S g n(\lambda-k)[G(t, x, \lambda, k)-G(t, x, k, \lambda)] \gamma(t) d P_{(t, x)}(\lambda) d \widehat{P}_{(t, x)}(k) d x d t$.

Proof. $I_{2}=\int_{(Q \times \mathbb{R})^{2}} S g n(\lambda-k)[G(t, x, \lambda, k)-G(s, y, k, \lambda)] \gamma(t) A_{n} d \nu d \widehat{\nu}=$ $\int_{(Q \times] 0,1[)^{2}}\{[G(t, x, u(t, x, \alpha), \hat{u}(s, y, \beta))-G(s, y, \hat{u}(s, y, \beta), u(t, x, \alpha))]$

$\left.\gamma(t) A_{n}(t, s, x, y) \operatorname{Sgn}(u(t, x, \alpha)-\hat{u}(s, y, \beta))\right\} d \alpha d \beta d x d t d y d s$.

Let us note

$$
\begin{aligned}
I_{2}^{\prime}= & \int_{(Q \times] 0,1[)^{2}}\{[G(t, x, u(t, x, \alpha), \hat{u}(t, x, \beta))-G(t, x, \hat{u}(t, x, \beta), u(t, x, \alpha))] \\
& \left.\gamma(t) A_{n}(t, s, x, y) \operatorname{Sgn}(u(t, x, \alpha)-\hat{u}(t, x, \beta))\right\} d x d t d y d s d \alpha d \beta .
\end{aligned}
$$

Thanks to the Lebesgue set properties of integrable functions and to the previous lemma, one shows that $\lim _{n \rightarrow \infty}\left|I_{2}-I_{2}^{\prime}\right|=0$ and concludes.

Step 3. $\lim _{n \rightarrow \infty} I_{3}=$

$-\int_{Q \times \mathbb{R}^{2}} \gamma(t) \operatorname{sign}(\lambda-k)[\operatorname{div} f(t, x, \lambda)-\operatorname{div} f(t, x, k)] d P_{(t, x)}(\lambda) d \widehat{P}_{(t, x)}(k) d t d x$.

Proof. $I_{3}=\int_{(Q \times \mathbb{R})^{2}} \gamma(t) \rho_{1}^{n}(t-s)[F(t, x, \lambda, k)-F(s, y, k, \lambda)] . \nabla_{x} \rho_{N}^{n}(x-$ y) $d \nu d \widehat{\nu}$.

Using,

$$
\begin{aligned}
& F(t, x, u(t, x, \alpha), \hat{u}(s, y, \beta))-F(s, y, u(t, x, \alpha), \hat{u}(s, y, \beta))= \\
& F(t, x, u(t, x, \alpha), \hat{u}(t, x, \beta))-F(s, y, u(t, x, \alpha), \hat{u}(t, x, \beta))+ \\
& F(t, x, u(t, x, \alpha), \hat{u}(s, y, \beta))-F(s, y, u(t, x, \alpha), \hat{u}(s, y, \beta))+ \\
& F(s, y, u(t, x, \alpha), \hat{u}(t, x, \beta))-F(t, x, u(t, x, \alpha), \hat{u}(t, x, \beta))
\end{aligned}
$$


one may write, $I_{3}=I_{3,1}+I_{3,2}+I_{3,3}$. Let us consider firstly $I_{3,1}$, then $I_{3,2}+I_{3,3}$.

i) $I_{3,1}=-\int_{(Q \times] 0,1[)^{2}} \gamma(t) \rho_{1}^{n}(t-s) \operatorname{sign}(u(t, x, \alpha)-\hat{u}(t, x, \beta))$

$[\operatorname{div} f(s, y, u(t, x, \alpha))-\operatorname{div} f(s, y, \hat{u}(t, x, \beta))] \rho_{N}^{n}(x-y) d t d x d \alpha d s d y d \beta-$ $\int_{Q \times \Sigma \times] 0,1\left[^{2}\right.}[F(t, x, u(t, x, \alpha), \hat{u}(t, x, \beta))-F(s, y, u(t, x, \alpha), \hat{u}(t, x, \beta))] \cdot \eta(y)$

$$
\gamma(t) \rho_{1}^{n}(t-s) \rho_{N}^{n}(x-y) d t d x d \alpha d s d \mathcal{H}^{n-1}(y) d \beta .
$$

$f$ is uniformly continuous on $\bar{Q} \times[-M, M]$ and thanks to lemma 10 i) and using the Lebesgue set properties of the $L^{1}$-functions, one shows that $I_{3,1}$ converges towards

$$
-\int_{Q \times] 0,1\left[\left[^{2}\right.\right.} \gamma(t) \operatorname{sign}(u(t, x, \alpha)-\hat{u}(t, x, \beta))
$$

$[\operatorname{div} f(t, x, u(t, x, \alpha))-\operatorname{divf}(t, x, \hat{u}(t, x, \beta))] d t d x d \alpha d \beta$.

ii) $I_{3,2}+I_{3,3}$. Let us note $\mathcal{S}$ the sign function.

As $F(t, x, u, \hat{u})-F(s, y, u, \hat{u})=\mathcal{S}(u-\hat{u})[f(t, x, u)-f(s, x, u)+$ $f(s, x, u)-f(s, y, u)-f(t, x, \hat{u})+f(s, x, \hat{u})-f(s, x, \hat{u})+f(s, y, \hat{u})]$, it comes that

$$
\begin{gathered}
F(t, x, u(t, x, \alpha), \hat{u}(s, y, \beta))-F(s, y, u(t, x, \alpha), \hat{u}(s, y, \beta)) \\
+F(s, y, u(t, x, \alpha), \hat{u}(t, x, \beta))-F(t, x, u(t, x, \alpha), \hat{u}(t, x, \beta))= \\
\int_{s}^{t} \mathcal{S}(u(t, x, \alpha)-\hat{u}(s, y, \beta))\left[\frac{\partial f}{\partial t}(\xi, x, u(t, x, \alpha))-\frac{\partial f}{\partial t}(\xi, x, \hat{u}(s, y, \beta))\right]- \\
\mathcal{S}(u(t, x, \alpha)-\hat{u}(t, x, \beta))\left[\frac{\partial f}{\partial t}(\xi, x, u(t, x, \alpha))-\frac{\partial f}{\partial t}(\xi, x, \hat{u}(t, x, \beta))\right] d \xi+ \\
\sum_{i=1}^{N} \int_{y_{i}}^{x_{i}} \mathcal{S}(u(t, x, \alpha)-\hat{u}(s, y, \beta))\left[\frac{\partial f}{\partial x_{i}}\left(s, \xi_{i}, u(t, x, \alpha)\right)-\frac{\partial f}{\partial x_{i}}\left(s, \xi_{i}, \hat{u}(s, y, \beta)\right)\right] \\
-\mathcal{S}(u(t, x, \alpha)-\hat{u}(t, x, \beta))\left[\frac{\partial f}{\partial x_{i}}\left(s, \xi_{i}, u(t, x, \alpha)\right)-\frac{\partial f}{\partial x_{i}}\left(s, \xi_{i}, \hat{u}(t, x, \beta)\right)\right] d \xi
\end{gathered}
$$


where, for $i \in\{1, . ., N\}$, we note $\xi_{i}=\left(x_{1}, . ., x_{i-1}, \xi, y_{i+1}, . ., y_{N}\right)$.

Using again the Lusin theorem (cf. lemma 10 ii)) and thanks to the following property of the sequence $\left(\rho_{N}^{n}\right)$ :

$\exists \digamma>0$, such that $\forall i \in\{1, . ., N\}, \forall n \in \mathbb{N}, \forall x \in \Omega, \forall t \in[0, T]$,

$$
\begin{gathered}
\int_{\Omega}\left|x_{i}-y_{i}\right| \cdot\left|\frac{\partial}{\partial x_{i}} \rho_{N}^{n}(x-y)\right| d y<\digamma \\
\int_{] 0, T[} \int_{\Omega}|t-s| \cdot\left|\frac{\partial}{\partial x_{i}} \rho_{N}^{n}(x-y)\right| \rho_{1}^{n}(t-s) d y d s<\digamma ;
\end{gathered}
$$

one has : $\lim _{n \rightarrow \infty} I_{3,2}+I_{3,3}=0$ and concludes.

Step 4. $\lim _{n \rightarrow \infty} I_{4}=0$.

Proof. Thanks to the fact that $\gamma$ belongs to $\mathcal{D}(0, T)$.

Step 5. $\lim _{n \rightarrow \infty} I_{5}=\frac{1}{2} \int_{\Sigma \times \mathbb{R}} F\left(t, x, \lambda, u^{B}\right) \cdot \eta \gamma(t) d \mu$.

Proof. Thanks to the fact that $\int_{Q} \rho_{1}^{n}(t-s) \rho_{N}^{n}(x-y) d s d y$ converges towards $\frac{1}{2}$ as $n$ goes to infinity.

In a similar way, one has : $\lim _{n \rightarrow \infty} I_{5}$ bis $=\frac{1}{2} \int_{\Sigma \times \mathbb{R}} F\left(s, y, k, \hat{u}^{B}\right) \cdot \eta \gamma(s) d \widehat{\mu}$.

Step 6. $\lim _{n \rightarrow \infty} I_{6}=\frac{1}{2} \int_{\Sigma \times \mathbb{R}} F\left(s, y, k, u^{B}(s, y)\right) \cdot \eta(y) \gamma(s) d \widehat{\mu}$.

Proof. $I_{6}=\int_{\Sigma \times \mathbb{R} \times] 0, \varepsilon[\times \Sigma} F\left(t, \bar{x}, k, u^{B}(t, \bar{x})\right) \cdot \eta(\bar{x}) \gamma(t) A_{n}$

$$
J(\bar{y}, \xi) d \mathcal{H}^{N}(t, \bar{x}) d \widehat{P}_{(s, y)}(k) d \xi d \mathcal{H}^{N}(s, \bar{y}) .
$$

Let $\delta>0$.

There exist a continuous function $\widetilde{u}^{B}$ on $\Sigma$ and a borelian $K, K \subset \Sigma$ with $\mathcal{H}^{N}(\Sigma \backslash K) \leq \delta$ and $\widetilde{u}^{B}=u^{B}$ on $K$.

Since $f$ is a bounded function on $\bar{Q} \times[-M, M]$, one can write $I_{6}=$ $I_{6}^{\prime}+o(\delta)$ where

$$
I_{6}^{\prime}=\int_{\Sigma \times \mathbb{R} \times] 0, \varepsilon[\times \Sigma} F\left(t, \bar{x}, k, \widetilde{u}^{B}(t, \bar{x})\right) \cdot \eta(\bar{x}) \gamma(t) A_{n}
$$


GUY VALLET DIRICHLET PROBLEM FOR A NONLINEAR CONSERVATION LAW ...

$$
J(\bar{y}, \xi) d \mathcal{H}^{N}(t, \bar{x}) d \widehat{P}_{(s, y)}(k) d \xi d \mathcal{H}^{N}(s, \bar{y}) .
$$

As $\operatorname{supp}(\gamma)$ is a compact set of $] 0, T\left[, F\left(t, \bar{x}, k, \widetilde{u}^{B}(t, \bar{x})\right) \gamma(t)\right.$ is uniformly continuous and $I_{6}^{\prime}$ has the same limit, when $n$ goes to infinity, as

$$
\begin{gathered}
I_{6}=\int_{\Sigma \times \mathbb{R} \times] 0, \varepsilon[\times \Sigma} F\left(s, y, k, u^{B}(s, \bar{y})\right) \cdot \eta(\bar{x}) \gamma(s) A_{n} \\
J(\bar{y}, \xi) d \mathcal{H}^{N}(t, \bar{x}) d \widehat{P}_{(s, y)}(k) d \xi d \mathcal{H}^{N}(s, \bar{y}) .
\end{gathered}
$$

Then, using a technique of local map ([14] and [9]), one shows that

$$
\lim _{n \rightarrow \infty} I "{ }_{6}=\frac{1}{2} \int_{\mathbb{R} \times \Sigma} F\left(s, y, k, \widetilde{u}^{B}(s, y)\right) \cdot \eta(y) \gamma(s) d \widehat{\mu} .
$$

Thus, the conclusion leads from

$$
\begin{aligned}
& \int_{Q \times \mathbb{R} \times \Sigma} F\left(s, y, k, \tilde{u}^{B}(s, y)\right) \cdot \eta(y) \gamma(s) d \widehat{\mu} \\
= & \int_{Q \times \mathbb{R} \times \Sigma} F\left(s, y, k, u^{B}(s, y)\right) \cdot \eta(y) \gamma(s) d \widehat{\mu}+o(\delta) .
\end{aligned}
$$

In a similar way, one has :

$$
\lim _{n \rightarrow \infty} I_{6} \text { bis }=\frac{1}{2} \int_{\Sigma \times \mathbb{R}} F\left(t, x, \lambda, \hat{u}^{B}(t, x)\right) \cdot \eta(x) \gamma(t) d \mu .
$$

Step 7. Conclusion. As we have

$$
\begin{gathered}
\left|F\left(t, x, u, u^{B}\right)+F\left(t, x, \hat{u}, \hat{u}^{B}\right)-F\left(t, x, u, \hat{u}^{B}\right)-F\left(t, x, \hat{u}, u^{B}\right)\right| \leq \\
2 \Delta\left(t, x, u^{B}, \hat{u}^{B}\right),
\end{gathered}
$$

it comes :

$$
\begin{gathered}
-\int_{Q} \gamma^{\prime}(t) \int_{\mathbb{R}^{2}}|\lambda-k| d P_{(t, x)}(\lambda) d \widehat{P}_{(t, x)}(k) d x d t+ \\
\int_{Q} \int_{\mathbb{R}^{2}} S g n(\lambda-k)[g(t, x, \lambda)-g(t, x, k)] \gamma(t) d P_{(t, x)}(\lambda) d \widehat{P}_{(t, x)}(k) d x d t \leq
\end{gathered}
$$




$$
\int_{\Sigma} \Delta\left(t, x, u^{B}, \hat{u}^{B}\right) \gamma(t) d \mathcal{H}^{N} .
$$

Since $u_{0}$ and $\hat{u}_{0}$ are initial conditions in the sense of definition 3 , for $t$ a.e. in $] 0, T[$,

$$
\begin{gathered}
\int_{\Omega \times \mathbb{R}^{2}}|\lambda-k| d P_{(t, x)}(\lambda) d \widehat{P}_{(t, x)}(k) d x+ \\
\int_{0}^{t} \int_{\Omega \times \mathbb{R}^{2}} \operatorname{Sgn}(\lambda-k)[g(s, x, \lambda)-g(s, x, k)] d P_{(s, x)}(\lambda) d \widehat{P}_{(s, x)}(k) d x d s \leq \\
\int_{0}^{t} \int_{\Gamma} \Delta\left(s, x, u^{B}, \hat{u}^{B}\right) d \mathcal{H}^{N-1} d s+\int_{\Omega}\left|u_{0}-\hat{u}_{0}\right| d x .
\end{gathered}
$$

Since $g$ is a Lipschitz function, one has,

Corollary 11. With the same hypothesis, for $t$ a.e. in $] 0, T[$ :

$$
\begin{gathered}
\int_{\Omega \times \mathbb{R}^{2}}|\lambda-k| d P_{(t, x)}(\lambda) d \widehat{P}_{(t, x)}(k) d x \leq \\
\left(\int_{0}^{t} \int_{\Gamma} \Delta\left(s, x, u^{B}, \hat{u}^{B}\right) d \mathcal{H}^{N-1} d s+\int_{\Omega}\left|u_{0}-\hat{u}_{0}\right| d x\right) e^{c(g) t} \\
\text { where } \Delta(t, x, A, B)=\underset{\substack{\operatorname{Sup} \\
\operatorname{Sin}(A, B) \leq c, d \leq \max (A, B)}}{|f(t, x, c)-f(t, x, d)| .}
\end{gathered}
$$

Corollary 12. There exists a unique EMVS to problem $(P)$, moreover this EMVS is the Young measure associated to an essentially bounded measurable function $u$ (i.e. a weak entropic solution).

Proof. If $u_{0}=\hat{u}_{0}$ and $u^{B}=\hat{u}^{B}$, then

$$
\int_{\Omega \times \mathbb{R}^{2}}|\lambda-k| d P_{(t, x)}(\lambda) d \widehat{P}_{(t, x)}(k) d x=0
$$

a.e. in $] 0, T$. Thus, the two measures $d P_{(t, x)}$ and $d \widehat{P}_{(t, x)}$ are equal to the Dirac measure centred in a point noted $u(t, x)$ (see [6] and [12]). Moreover, $u$ is a measurable function, essentially bounded by $M$, the boundary of the support of the measure $d P_{(t, x)}$. Then, the EMVS is unique and it is the Young measure associated to $u$. 
Corollary 13. Assume that $u$ and $\widehat{u}$ are two weak entropic solutions to problem $(P)$ with two initial conditions $u_{0}$ and $\hat{u}_{0}$ and two boundary conditions $u^{B}$ and $\hat{u}^{B}$. Then for $t$ a.e. in $] 0, T[$ :

$$
\begin{gathered}
\|u(t)-\hat{u}(t)\|_{L^{1}(\Omega)} \leq \\
\left(\int_{0}^{t} \int_{\Gamma} \Delta\left(s, x, u^{B}, \hat{u}^{B}\right) d \mathcal{H}^{N-1} d s+\left\|u_{0}-\hat{u}_{0}\right\|_{L^{1}(\Omega)}\right) e^{c(g) t} .
\end{gathered}
$$

If $f$ is a Lipschitz function with respect to its third variable, then for $t$ a.e. in $] 0, T[$ : one has :

$$
\begin{gathered}
\|u(t)-\hat{u}(t)\|_{L^{1}(\Omega)} \leq \\
\left(c(f)\left\|u^{B}-\hat{u}^{B}\right\|_{L^{1}(\Sigma)}+\left\|u_{0}-\hat{u}_{0}\right\|_{L^{1}(\Omega)}\right) e^{c(g) t} .
\end{gathered}
$$

\section{References}

[1] E.J. Balder, Lectures on Young Measures, cahiers de mathématiques de la décision du CEREMADE, $\mathrm{n}^{\circ} 951$ (1995).

[2] C. Bardos, A.Y. Leroux, J.C. Nedelec, First order quasilinear equations with boundary conditions, Comm. in P.D.E., 4 (9) (1979), 1017-1034.

[3] C. Chainais-Hillairet, Finite volume schemes for a nonlinear hyperbolic equation. Convergence towards the entropy solution and error estimate, RAIRO-M²AN, Vol. 33, nº1 (1999), 129-156.

[4] S. Champier, Error estimates for the approximate solution of a nonlinear hyperbolic equation with source term given by finite volume scheme, Publication $n^{\circ} 259$ de l'UMR-5585-Lyon/Saint-Etienne (1997).

[5] B. Cockburn, F. Coquel, P.G. LeFloch, Convergence of the finite volume method for multidimensional conservation laws, SIAM J. Numerical Anal., 32, $\mathrm{n}^{\circ} 3$ (1995), 687-705.

[6] R. Di perna, Measure-valued solutions to conservation laws, Arch. Rat. Mech. Anal., 88 (1985), 223-270.

[7] R. Eymard, Th. Gallouët, R. Herbin, Existence and uniqueness of the entropy solution to a nonlinear hyperbolic equation, Chin. Ann. of Math., Vol. 16 B:1 (1995), 1-14. 
[8] L. Lévi, Modélisation par des problèmes hyperboliques de perturbations d'écosystèmes hydriques, Thèse de l'Université de Pau et des Pays de l'Adour, 1994.

[9] F. Otto, Conservation laws in bounded domains, uniqueness and existence via parabolic approximation, Chap. 2 §.2.6, $2.7 \&$ 2.8, pp.95-143 in Malek J., Necas J., Rokyta M., Ruzicka M., Weak and measure-valued solutions to evolutionary PDE's, Chapman\&Hall, 1996.

[10] J.P. Penot, M. Volle, Inversion of real-valued functions and applications, ZOR, Methods and Models of Operations Research, 34 (1990), 117-141.

[11] A. Szepessy, Measure-valued solution of scalar conservation laws with boundary conditions, Arch. Rat. Mech. Anal., Vol. 107, n ${ }^{\circ} 2$ (1989), 182193.

[12] L. Tartar, Compensated compactness and application to partial differential equations, Nonlinear Analysis and Mechanics, Heriot-Watt Symposium, Vol. IV, Research Notes in Mathematics, 39, Pitman, London (1979), 136-192.

[13] M. Valadier, A course on Young Measures, Workshop di teoria delle misura e analisi reale, Grado, September 19 October 2, 1993, Rend. Istit. Mat. Univ. Trieste, 26 suppl. (1994), 349-394.

[14] G. Vallet, Uniqueness of the measure-valued solution to a conservation law with boundary conditions, Appl. Math. Lett., vol.11, n4 (1998), 91-94.

[15] G. Vallet, A contribution to the study of a first-order non-linear hyperbolic equation with boundary conditions, Publication interne du Laboratoire de Mathématiques Appliquées de l'Université de Pau UPRES-A CNRS 5033, $\mathrm{n}^{\circ} 98 / 27$ (1998).

[16] M.H. Vignal, Schémas volumes finis pour des équations elliptiques ou hyperboliques avec conditions aux limites, convergence et estimations d'erreurs, Thèse de l'Ecole Normale Supérieure de Lyon, 1997.

I.P.R.A. CNRS ERS 2055

Av. de l'Université 64000

Pau (France)

E-mail address: guy.vallet@univ.pau.fr

Recibido: 9 de Abril de 1999

Revisado: 19 de Enero de 2000 